\title{
Study on Dahlin algorithm of Brushless DC motor based on Neural
}

\author{
Network \\ ZILONG HUANG ${ }^{1}$, DAN WANG ${ }^{2}$, LELE XI ${ }^{3}$, YANKAI SHEN ${ }^{4}$ \\ ${ }^{1}$ School of electrical engineering ,Hebei University of Science and Technology, Shijiazhuang, China \\ 2 School of electrical engineering, Hebei University of Science and Technology, Shijiazhuang, China \\ aE-MAIL:837384556@qq.com, ${ }^{\mathrm{b} E-M A I L: 935830394 @ q q . c o m ~}$ \\ cE-MAIL:532373798@qq.com ,dE-MAIL:shenyankai1989@163.com
}

Keywords: brushless DC motor Dahlin control neural network

\begin{abstract}
Brushless DC motor has the advantages of simple structure, reliable operation, convenient maintenance, high efficiency, no excitation loss and good speed regulation performance. Dahlin algorithm is to find a reasonable digital regulator $D(\mathrm{z})$, which can make the closed-loop transfer function of the system is equivalent to a combination of an inertia link and a delay link, to achieve a good control effect.Brushless DC motor is a multi variable, strongly coupled nonlinear system, and the fixed parameters of Dahlin regulator can not get ideal control performance index. In this paper, as the control object, the brushless DC motor is based on the BP neural network, and the Dahlin controller based on neural network for brushless DC motor is designed.
\end{abstract}

\section{Introduction}

It is difficult to control the time varying delay of the control object, and it is one of the subjects in control field that controlling the time varying delay. Dahlin controller is a frequently-used controller which is used in the lagging system control field. The Dahlin algorithm used in time delay system needs to choose the appropriate sampling period and the time constant of the closed-loop system, so the algorithm can only be used for the time-delay control system with constant ${ }^{[1]}$. Artificial neural network has a high capacity of parallel distributed processing, through the study of the input and output sample, it can constantly adjust the network weights composed of multiple neuron models, and realize the mapping relationship between the input and output, and has the ability of learning, memory, computing and all kinds of intelligent processing ${ }^{[2]}$. In this paper, a control method based on BP neural network is proposed, by using neural network to study the output of Dahlin controller, the adaptive stabilization control of time delay system can be realized.

\section{The method of Dahlin controller based on neural network}

\subsection{The basic principle of Dahlin algorithm}

The design goal of Dahlin algorithm is to find a reasonable digital regulator $D(\mathrm{z})$, which can make the closed-loop transfer function of the system is equivalent to a combination of an inertia link and a delay link,and the lag time of both closed-loop control system and controlled object is required to be equal ${ }^{[3]}$.Dahlin algorithm is relatively simple in theory, only to find a reasonable and achievable digital regulator $D(\mathrm{z})$, it can effectively eliminate the adverse effects caused by the delay,and reasonable adjustment parameters can achieve the requirement of the overshoot and the 
reaction rate.For example,the controlled object is abstracted as:

$$
G_{c}(s)=\frac{K}{1+T_{1} s} e^{-\tau s} .
$$

Among them, $\tau$ is the lag time; $T_{1}, K$ are the amplification factors.

The design goal of Dahlin algorithm is to make the transfer function $\phi(s)$, which is expected to the whole closed-loop system, equivalent to a delay link and an inertia link in series, that is,

$$
\phi(s)=\frac{1}{1+T_{\tau} s} e^{-\tau s} .
$$

And it requires that the lag time both the closed loop control system and the controlled object is equal. In the formula (2), $T_{\tau}$ is the time constant of the closed-loop system,Lag time $\tau$ has an integer multiple with the sampling period $\mathrm{T}$ :

$$
\tau=N T \quad(N=1,2,3,4 \cdots \cdots)
$$

Using the impulse transfer function approximation method to obtain the closed loop pulse transfer function $\phi(z)$, which is corresponding to $\phi(s)$, it is :

$$
\phi(z)=\frac{Y_{(z)}}{G_{(z)}}=z\left[\frac{1-e^{-T s}}{s} \cdot \frac{e^{-\tau s}}{T_{\tau} s+1}\right] .
$$

Bring $\tau=T N$ into the formula (4), and do $\mathrm{Z}$ transform, we can get:

$$
\phi(z)=\frac{\left(1-e^{-T / T_{\tau}}\right) z^{-N-1}}{1-e^{-T / T_{\tau}} z^{-1}} .
$$

So,we can get:

$$
D(z)=\frac{1}{G(z)} \cdot \frac{\phi(z)}{1-\phi(z)}
$$

If the pulse transfer function $G(\mathrm{z})$ of the controlled object is known, the pulse transfer function $D$ (z) of the digital controller can be obtained by the formula (6).

Dahlin algorithm for a first-order inertial with lag is:

$$
\begin{aligned}
& G(z)=Z\left[\frac{1-e^{-\tau s}}{s} \cdot \frac{k e^{-\tau s}}{1+T_{1} s}\right]=k z^{-N-1} \frac{1-e^{-T / T_{1}}}{1-e^{-T / T_{1}} z^{-1}} . \\
& D(z)=\frac{1}{G(z)} \cdot \frac{z^{-N-1}\left(1-e^{-T / T \tau}\right)}{1-e^{-T / T \tau} z^{-1}-\left(1-e^{-T / T \tau}\right) z^{-N-1}} .
\end{aligned}
$$


Bring $G(z)$ into the formula (8),we can get:

$$
D(z)=\frac{\left(1-e^{-T / T_{1}} z^{-1}\right)\left(1-e^{-T / T_{\tau}}\right)}{k\left(1-e^{-T / T_{1}}\right)\left[1-e^{-T / T_{\tau}} z^{-1}-1-e^{-T / T \tau} z^{-N-1}\right]} .
$$

The expression can be deformed to:

$$
D(z)=\frac{a-b z^{-1}}{c-d z^{-1}-e z^{-2}} .
$$

Among them, $\mathrm{a}$ and $\mathrm{b}$ are the control parameters.

2.2 The basic principle of BP neural network

The neural network is not good at expressing knowledge by explicit formulation, but it has a very strong ability to approximate nonlinear function, that is, the ability of nonlinear mapping. The neural network is used to control just because of this unique advantage, and in fact, it is a reverse process for the controlled object ${ }^{[4]}$. Neural network directly acts as the controller in the feedback control system, its structure is shown in figure 1.

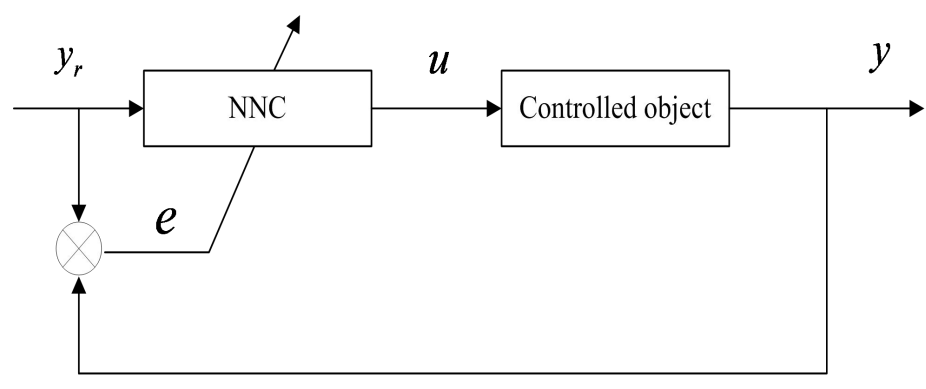

Figure 1. Neural network feedback system control structure diagram

BP neural network is one of the most extensive and applied models in artificial neural network, its structure is shown in Figure 2. $\mathrm{x}$ and $\mathrm{z}$ are the input and output vector of the network, each neuron is represented by a node, the network is composed of an input layer, a hidden layer and an output layer, the hidden layer can be a layer or more layers, and the front layer node to the back layer node is connected by the weight coefficient. The input signal of BP neural network from the input layer to the output layer through the hidden layer(forward propagation), if the output layer gets the desired output, then the learning algorithm ends; Otherwise, go to the back propagation. Back propagation is to reverse calculation error signal according to the original connection pathway, and adjust the weights of the neurons by the gradient descent method, so that the error can be reduced ${ }^{[5,6]}$.

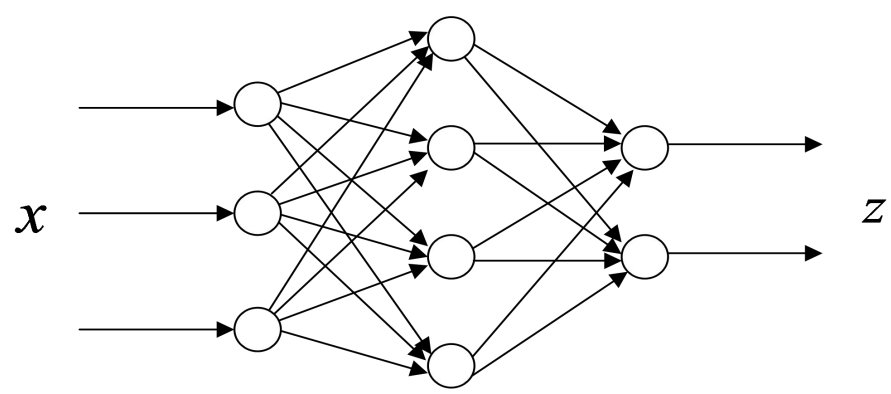

Figure 2.The structure of BP neural network

2.3 Dahlin control method based on Neural Network 
BP neural network has the ability of approximate arbitrary nonlinear function, and the structure and learning algorithm is simple and clear. The feedback system control structure diagram of Dahlin control method based on neural network is shown in figure 3.

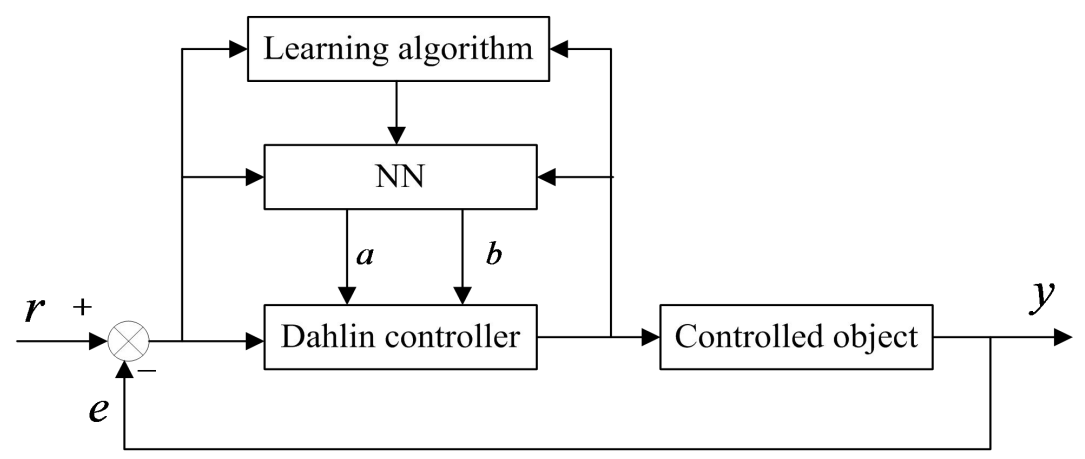

Figure 3. The feedback system control method based on Neural Networ method based on neural network

Through the study of the network itself, we can find the initial values of the Dahlin parameters in the optimal control law, and after that, neural network will learn adjustment. The structure of Dahlin control method based on Neural Network is shown in figure 4.

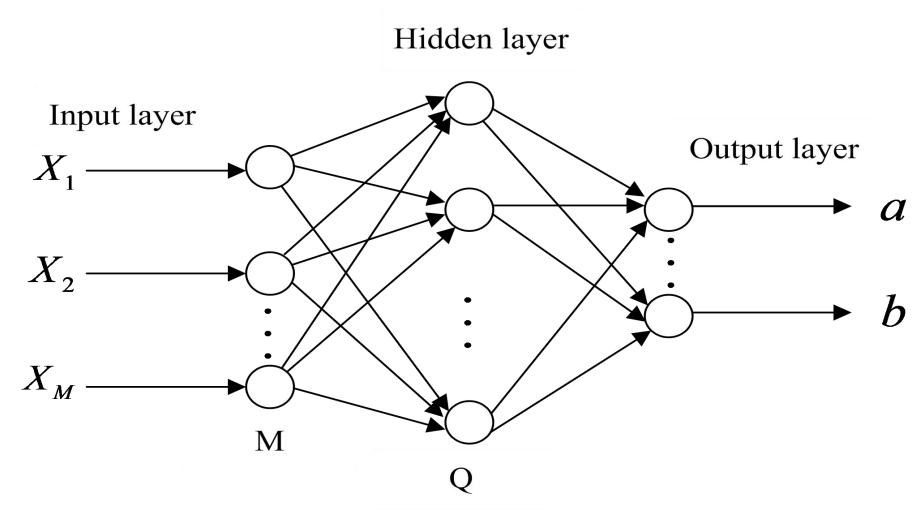

Figure 4. The structure of Dahlin control structure diagram of Dahlin control

Therefore, the mathematical algorithm with weight coefficient of the NN output layer of BP neural network can be obtained :

$$
\begin{aligned}
& \Delta \omega_{l i}^{(3)}(k)=\eta \delta_{l}^{(3)} O_{l}^{(2)}(k)+\alpha \Delta \omega_{l i}^{(3)}(k-1) \\
& \delta_{i}^{(3)}=e(k) \operatorname{sgn}\left(\frac{\partial y(k)}{\partial \Delta u(k)}\right) \frac{\partial \Delta u(k)}{\partial O_{l}^{(3)}(k)} g^{\prime}\left(n e t_{l}^{(3)}(k)\right) . \quad(l=1,2,3)
\end{aligned}
$$

According to the above calculation method, the mathematical method of the weight coefficient of the hidden layer is:

$$
\begin{aligned}
& \Delta \omega_{i j}^{(2)}(k)=\eta \delta_{i}^{(2)} O_{j}^{(1)}(k)+\alpha \Delta \omega_{i j}^{(2)}(k-1) . \\
& \delta_{i}^{(2)}=f^{\prime}\left(n e t_{i}^{(2)}(k)\right) \sum_{l=1}^{3} \delta_{l}^{(3)} \omega_{l i}^{(3)}(k) . \quad(i=1, \ldots, Q)
\end{aligned}
$$

In the formula, $g^{\prime}[\bullet]=g(x)[1-g(x)] . \quad f^{\prime}[\bullet]=\left[1-f^{2}(x)\right] / 2$. 
The neural network learning is carried out by the above algorithm, and the weighted coefficient is adjusted online, and the adaptive adjustment of the parameters of the Dahlin control is realized.

\section{Simulation application of Dahlin algorithm system for brushless DC motor based on Neural Network}

The Dahlin control algorithm based on BP neural network is used to simulate the nonlinear system with time-varying parameters, at the same time, compared with the ordinary Dahlin control algorithm, can be fully reflected that Dahlin control algorithm based on neural network is adaptive to environmental change, strong robustness, better control characteristics and overcome the shortcomings of parameters of the traditional Dahlin controller, which can not be well adapted to the fluctuations of the system.

In this paper, the Matlab/Simulink software is used to simulate the brushless DC motor. The actual parameters of the brushless DC motor are shown in Table 1.

Table 1. The actual parameters of Brushless DC motor

\begin{tabular}{|ll|}
\hline Parameter name & Parameter values \\
\hline Rated speed & $3000(\mathrm{r} / \mathrm{min})$ \\
Electromotive force coefficient & $0.112(\mathrm{~V} /(\mathrm{rad} / \mathrm{s}))$ \\
\hline Winding resistance & $1(\Omega)$ \\
\hline Self-induction & $2.2(\mathrm{mH})$ \\
Mutual inductance & $0.69(\mathrm{mH})$ \\
\hline Moment of inertia & $2.5 \times 10^{-5}\left(\mathrm{~kg} \cdot \mathrm{m}^{2}\right)$ \\
\hline
\end{tabular}

(1) The rapidity and stability of the system

Motor speed at the rated operating state is $3000 \mathrm{r} / \mathrm{min}$. Simulation results are shown in Figure 5, the simulation results show that when the system meets the requirements of overshoot,compared to pure Dahlin control, the Dahlin control system based on BP neural network has better performance, stability and saturation.

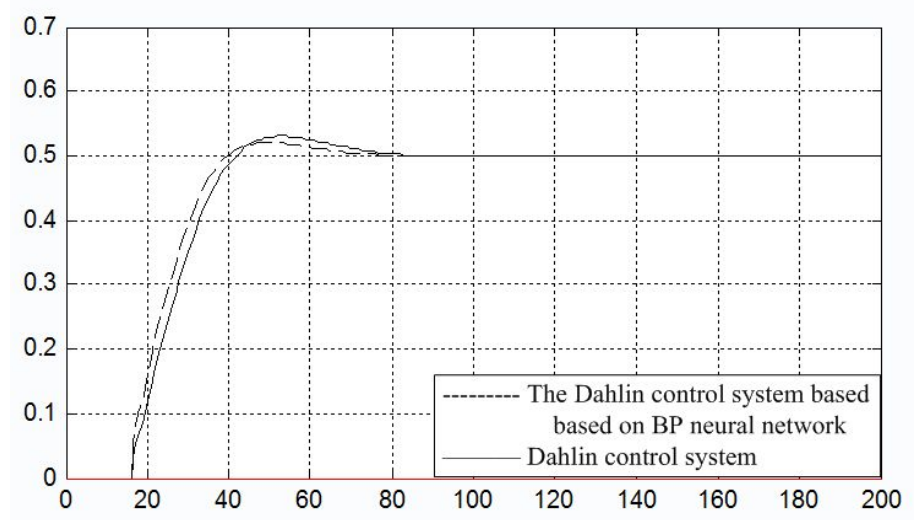

Figure 5. Simulation curves at the rated operating state

(2) Effect of motor parameter variation on system robustness

When the moment of inertia is doubled, the speed curve is shown in Figure 6 and 7. The simulation results show that the Dahlin controller based on BP neural network has the strongest robustness. 


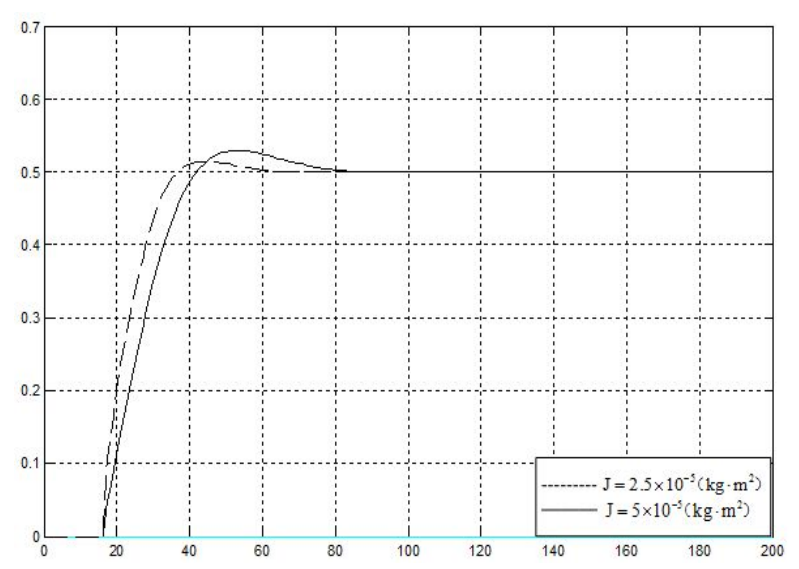

Figure 6. Simulation curve of Dahlin algorithm controller

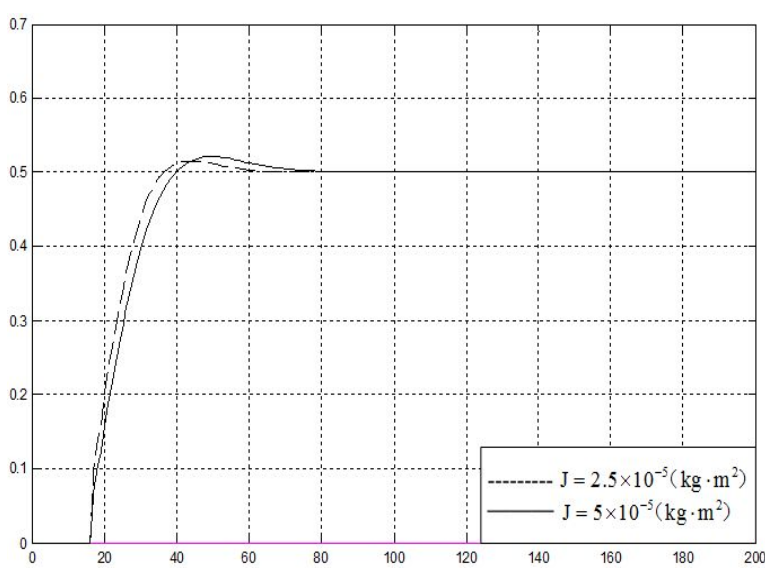

Figure 7. Simulation curve of Dahlin controller based on BP neural network

\section{Conclusions}

The algorithm of the speed controller of Brushless DC motor servo system based on BP neural network Dahlin controller is a kind of algorithm with self-learning and adaptive ability, it has the advantages of simple structure, clear physical meaning, small amount of computation, strong robustness and anti-interference ability. The neural network algorithm is combined with the Dahlin controller, so that the controller parameters can be adjusted easily and can adapt to the complex environment. Simulation results are demonstrated by comparing the Dahlin controller based neural network and the Dahlin controller, the system has the advantages of fast response, small overshoot. And the parameters in the Dahlin controller based neural network can be self-turning, brushless DC motor controller has high stability when load disturbance is high.

\section{References}

[1] Dahlin E B.Designing and tuning digital controller[J].Inatru and Control Systems, 1968,41(6):77-83.

[2] Han Liqun. Design and application of artificial neural network theory. Beijing: Chemical Industry Press, 2002.

[3] Wen Dingdu. Study on Dahlin control of neural network for pure delay system[J]. Automatic instrumentation, 2008,29 (12): 38-43.

[4] Tan Yonghong. Adaptive control based on BP neural network[M]. Control theory and applicationsx.1994,11 (1): 84-87.

[5] Xu Lina. Neural network control [M]. Beijing: Electronic Industry Press, 2003.

[6] He Yubin Li, Xinzhong. Neural network control technology and its application. Science Press, 2000 . 